\title{
Three rare variants of SOX7 impairing its interaction with GATA4 may be a predisposing factor to complete AVSD
}

\author{
Baolei $\mathrm{Li}^{1}$, Zhuoyan $\mathrm{Li}^{1}$, Jianping Yang ${ }^{1}$, Nan chao Hong ${ }^{1}$, Lihui Jin ${ }^{1}$, Yue-Juan Xu${ }^{1}$, \\ Qihua $\mathrm{Fu}^{2}$, Kun $\mathrm{Sun}^{1}$, Yu Yu${ }^{1}$, Yanan $\mathrm{Lu}^{1}$, and Sun Chen ${ }^{1}$ \\ ${ }^{1}$ Shanghai Jiaotong University School of Medicine Xinhua Hospital \\ ${ }^{2}$ Shanghai Childrens Medical Center Affiliated to Shanghai Jiaotong University School of \\ Medicine
}

April 28, 2020

\begin{abstract}
Atrioventricular septal defects (AVSD) are a complicated subtype of congenital heart defects for which the genetic basis is poorly understood. Many evidences have demonstrated that transcription factor SOX7 which can interact with GATA4 plays a pivotal role in the cardiovascular development. The critical role of GATA4 in the morphogenesis of atrioventricular septum implies SOX7 a potential involvement of AVSD. However, whether SOX7 variants are involved in the pathogenesis of AVSD needs to be explored. Through target sequencing, we identified three rare variants c.40C $>\mathrm{G}$, c.542G $>\mathrm{A}$, and c.743C $>\mathrm{T}$ of $\mathrm{SOX} 7 \mathrm{in} 100$ sporadic non-syndromic AVSD Chinese Han patients. All mutant sites were highly conserved in mammals. The mRNA and protein levels of SOX7 variants were altered compared with those of the wildtype. Moreover, SOX7 overexpression promoted the expression of GATA4 in human umbil-ical vein endothelial cells. Chromatin immunoprecipitation assay uncovered that SOX7 could directly bind to the region of GATA4 promoter. Luciferase assays demonstrated that SOX7 activated GATA4 promoter and the variants impaired the transcriptional activity of SOX7. Furthermore, the variants of SOX7 altered the regulation to the activity of GATA4 on its target genes. Our studies provide evidence that deleterious variants in SOX7 are potential contributors to human AVSD and provide novel insights into the etiology of AVSD.
\end{abstract}

\section{Hosted file}

Main text Three rare variants of SOX7 imparing its interaction with GATA4 may be a predisposing factor available at https://authorea.com/users/314456/articles/444810-three-rare-variants-of-sox7impairing-its-interaction-with-gata4-may-be-a-predisposing-factor-to-complete-avsd 
figures/Tu-Pian-1/Tu-Pian-1-eps-converted-to.pdf 
figures/Tu-Pian-2/Tu-Pian-2-eps-converted-to.pdf 
figures/Tu-Pian-3/Tu-Pian-3-eps-converted-to.pdf 
figures/Tu-Pian-4/Tu-Pian-4-eps-converted-to.pdf 
figures/Tu-Pian--5/Tu-Pian--5-eps-converted-to.pdf 
figures/Tu-Pian--6/Tu-Pian--6-eps-converted-to.pdf 
figures/Tu-Pian-7/Tu-Pian-7-eps-converted-to.pdf 
figures/Tu-Pian-8/Tu-Pian-8-eps-converted-to.pdf 